\title{
A Design Method Based on Inherent Testability of Remote Control and Monitoring System for Marine Main Engine
}

\author{
Xuedong Wen ${ }^{1, a^{*}}$, Guo He ${ }^{2, b}$ and Jianbo $\mathrm{Li}^{3, \mathrm{c}}$ \\ ${ }^{1}$ College of Power Engineering, Naval Univ. of Engineering, Wuhan 430033,China \\ ${ }^{2}$.Department of Management Science, Naval Univ. of Engineering, Wuhan 430033,China \\ 3. Military Representative Office of Jiangnan Shipyard,Shanghai 200011, China

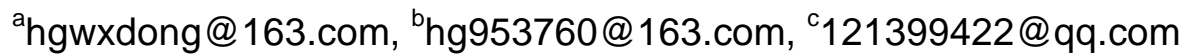

\begin{abstract}
Keywords: marine main engine, monitoring and control system, LRU, SRU, CAN bus
Abstract. During the design phase of a new electronic monitoring and control system, carrying out research on inherent testability can promote the entire system's testability level and make it be apt to diagnose fault and maintain high reliability. To improve the current insufficient application of inherent testability for remote control and monitoring system (RCMS) of marine main engine, this paper proposes a method to design RCMS, using microcomputer control and controller area network (CAN) technology, which bases on the perspective of the inherent testability .Firstly, the paper introduces the object oriented hierarchy structure of framework and three relatively independent subsystems are included: remote control sub-system (RCS), safety protection sub-system (SPS) as well as monitoring and alarm sub-system (MAS). And then, for each subsystem, functional unit division and module design are carried out, that is, conducting designs for Line Replace Unit (LRU) and Shop Replace Unit (SRU). Finally, dual redundant CAN bus accomplish the system network integration. After a long period time of ship test, RCMS has been running smoothly, and the experimental results meet the testability requirements.
\end{abstract}

\section{Introduction}

Design of Inherent testability is the basis of testability design and comprehensive testing capabilities for equipment [1]. Inherent testability refers to a design measure which is dependent only upon hardware design and is independent of test stimulus and response data $[2,3]$. It is convenient for fault detection and fault isolation. Besides, it supports both external test and built-in test. Thus, during a design process for a new equipment, to design and analysis the inherent testability can reduce life cycle costs and improve availability as well as diagnosis efficiency for the equipment.

With the development of the automation technology for modern ships, the level of design and function of RCMS for marine main engine has made great progress, but the testing and diagnosing problems for system problems attendant increasingly prominent. Currently, almost no research papers discuss the outcomes of RCMS From the point of inherent testability design and analysis, which result in a poor testability level, long testing time if failure, and a low rate of test accuracy.

In this paper, we propose a related research based on design for Inherent testability, use microcomputer control and CAN technology, and follow the $\square$ class control requirements for ship power plant monitoring system[4] to conduct a new design flow for RCMS. Using system-level division, LRU functional unit division and unit under test (UUT) module design, RCMS is organized with different serialization and standardization units, and dual redundant CAN network integrates the entire system and complete data exchange among UUTs. This research of the paper improve the inherent testability level for the whole RCMS of marine main engine.

\section{Processes of Inherent Testability Analysis and Application}

The main purpose of inherent testability is mainly for the fault detection and fault isolation, but affecting factors for the development of equipment testing programs are very complicated. According 
to the testability requirements, the procedures of RCMS based on inherent testability are referred with fig.1.

Refer to historical data, such as relevant monitoring systems' existing standards, experts' experience, and similar equipment failure modes and effects analysis, the inherent test set is initially set. And then we select the inherent weaknesses in the inherent test set through testability checklists and quantitative methods for evaluating. After the preliminary assessment of inherent test, a redesign of RCMS for a perfect designing testability is executed. Finally, the design elements constitute the monitoring system integrated with the design of inherent testability. Thus, the design process of based on inherent testability needs to be repeated iteration, which will make the RCMS gain the needs of test controllability, test observability and good compatibility features.

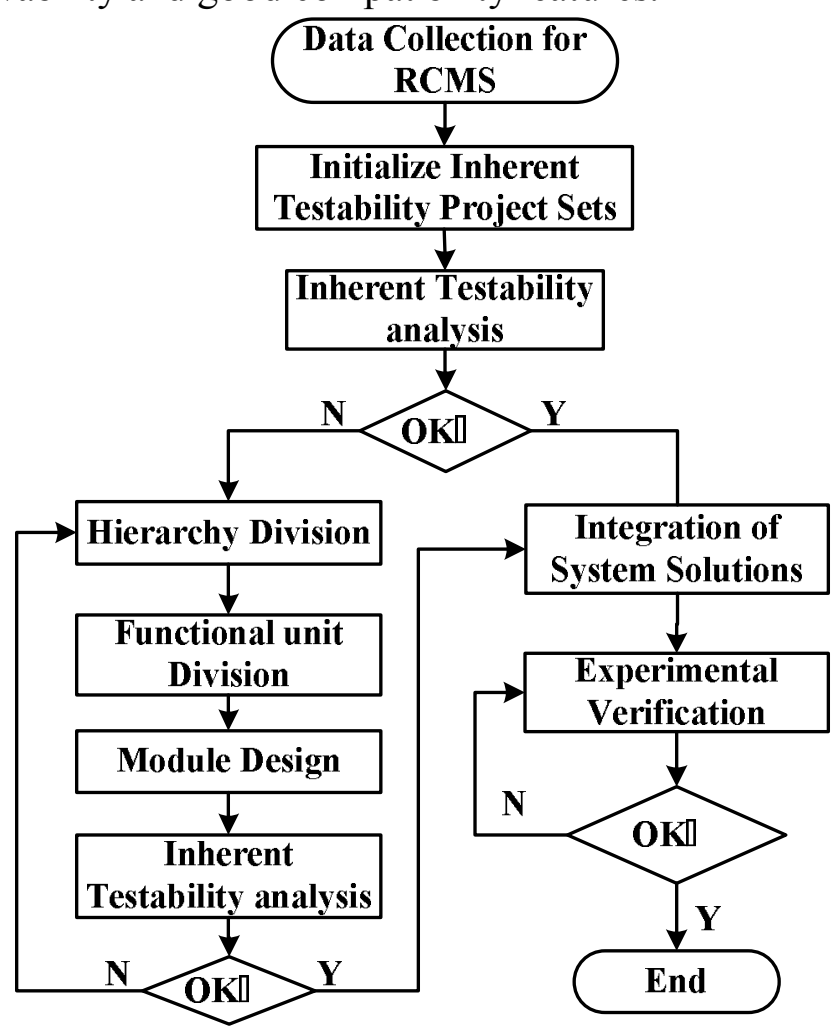

Fig. 1 The flow chart of inherent testability analysis and design for RCMS

\section{Division of Hierarchy Structure for RCMS}

A marine power plant equipped with two high-speed diesel engines use twin propellers to propel. And each diesel engine drives a fixed pitch propeller through a fluid coupling. The monitoring parameters associated with the power plant are about more than two hundred, and the control parameters are over one hundred. Because of so many monitoring and control nodes, the monitoring parameters are in addition to displaying on the screen of liquid crystal display (LCD) of industrial personal computer (IPC), and are also displayed on the console panel with lights, instruments, etc.

According to the own characteristics of RCMS, the paper apply the method of hierarchical design and divide the system into centralized management layer (CML), network control layer (NCL), and field device layer (FDL), refer with Fig. 2. Then, each layer can be further subdivided into a number of LRUs and SRUs. In the CML, the function of centralized operation and management are completed by hybrid engine telegraph (HET) and remote operating panel (ROP), and the function of alarm are demonstrate by monitoring and alarm panels, security dashboard and two mutually redundant IPC. The NCL accomplishes the process control operation, field data collection and ensure the system security. At the bottom layer of RCMS is the FDL which is constituted by the most critical devices, such as various sensors, electrical and hydraulic actuators, etc. the devices execute control commands of NCL the control layer, While providing on-site information to CML through routers. 


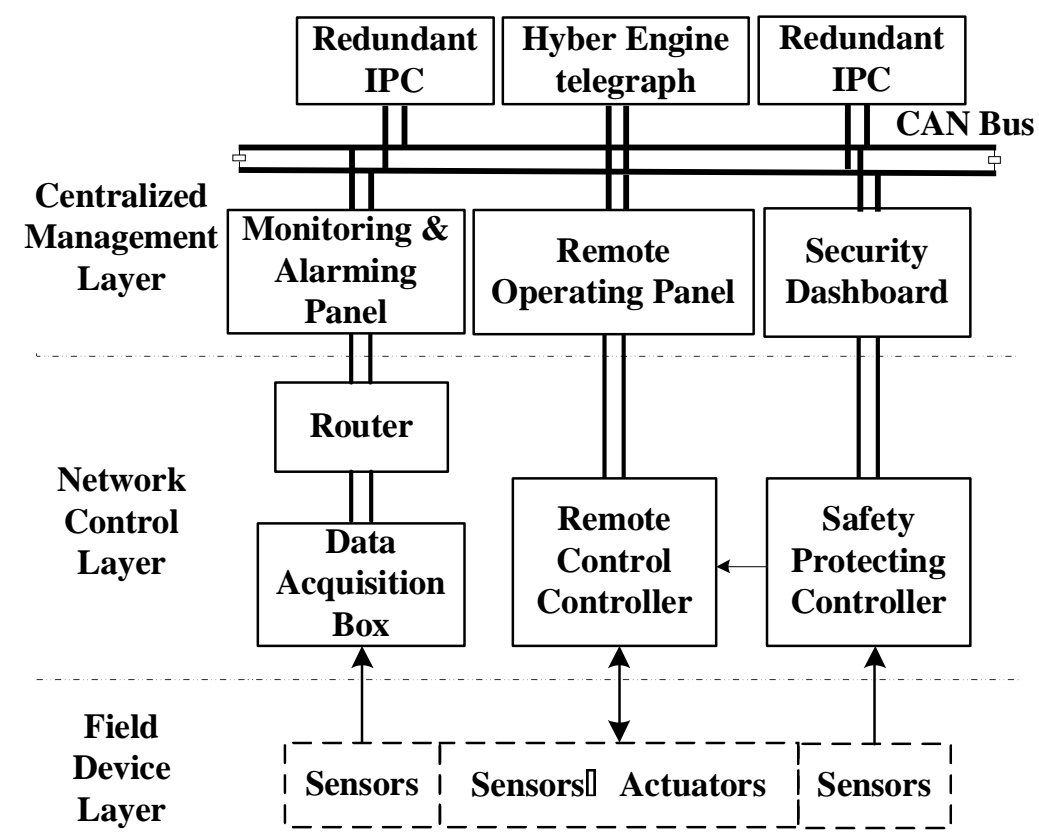

Fig.2 The Structure Diagram of a Marine Power Plant

\section{Structure Design of RCMS for Functional Units and Modules}

Functional unit division is a design process for LRU and is also the foundation of modular design and packaging for SRU. By functional division of LRU, it's better to design a single function into an individual interchangeable unit, which guarantee each functional fault can be individually tested and isolated. This article divides the surveillance system into three functional subsystems, RCS, SPS and MAS. All subsystems are interrelated and also relatively independent. Any sub-system is damaged does not affect the other subsystems, so the collapse risk of system is dispersion.

To refine and optimize the compositions of LRU and SRU is the main purpose by the module structure design. The process needs to consider UUTs with mass, volume, complexity, and linkages and other factors in order to facilitate the operations of fault detection, isolation, and replacement. The mainly key components of UUTs in the NCL are controllers and data acquisition modules. Controllers are responsible for the instruction execution process of RCS and SPS. The function of data acquisition module (DAM) collects running status signals of main power plant for MAS.

Remote Control Sub-System and Safety Protection Sub-System. Although RCS and SPS have separate hardware devices, they are shared in terms of some resources. For example, the function of start interlock protection in SPS is through remote controlling controller (RCC) in RCS to limit starting operation of a diesel engine (Fig. 2). Therefore, This article describes two subsystems together, and the functional relationship of two subsystem are referred with Fig.3.

RCS consists of several UUTs, such as hybrid engine telegraph, remote operating panel, remote display board, RCC, electronic speed regulation module, and electrical / hydraulic actuators, sensors, etc. Among them, Hybrid engine telegraph is responsible for liaison and information control. Remote operating panel send host remote control instructions. As the core control unit, RCC receive instructions from hybrid engine telegraph and remote operating panel, control operations of diesel engine's starting, stopping and running after integrated post-processing, and Upload status signals to the remote display board and IPC.

SPS consists of security alarming panels, safety protecting controller (SPC), signal sampling modules and sensors. Signal input module collects key parameters affecting system security from independent sensors of SPS. After receiving the sensor signal, SPC determines status of field devices and send instructions including monitoring,start interlock, fault slowly down or fault parking, and so on. At the same time, the instructions are transmitted to RCC or security alarming panels for further processing and display. 


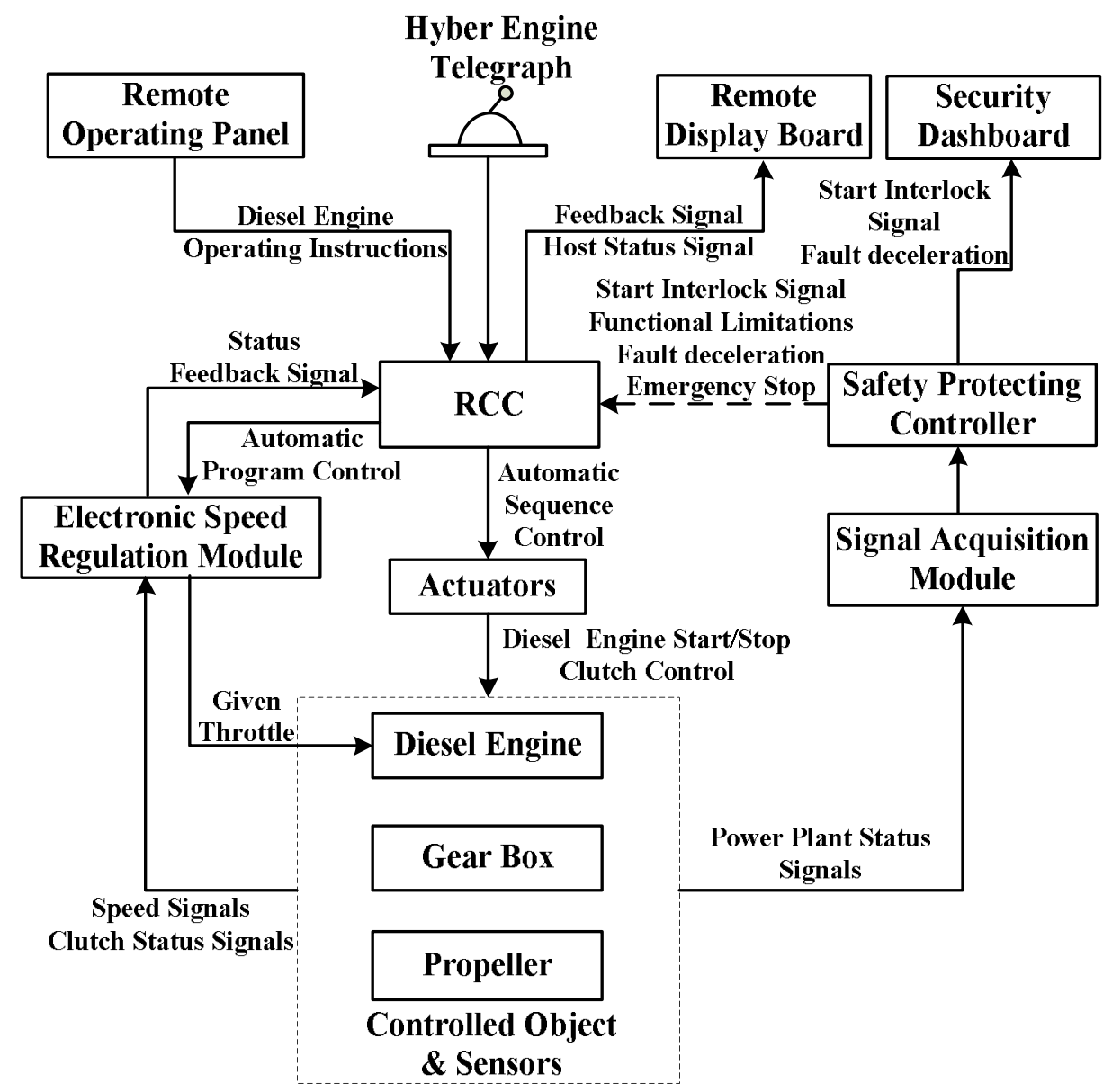

Fig. 3 The Organizational Chart of Remote Control Sub-system and Safety Protection Sub-system Monitoring and Alarm Sub-System. MAS mainly makes up of monitoring and alarm panels, routers, data acquisition box, and kinds of sensors. Data collection box is actually a distributed data acquisition and processing station, installed inside of types of standardized single-function data acquisition modules.

According to the characteristics of the monitoring objects' distribution and number, each of data acquisition box is equipped with different types and amounts of standardized measurement. Every measurement channel of signal acquisition module is connected with the corresponding sensor.

After processing sampled data, signal acquisition module sends the data to through the lower level Fieldbus CAN network. And then, the data is uploaded by the router and transferred to a monitoring and management computer and monitoring and alarm panels through the upper field bus. At last, the real-time function of monitoring thermal parameters and transferring alarm information for the power plant are realized. The basic functional organization of MAS refers with fig. 4.

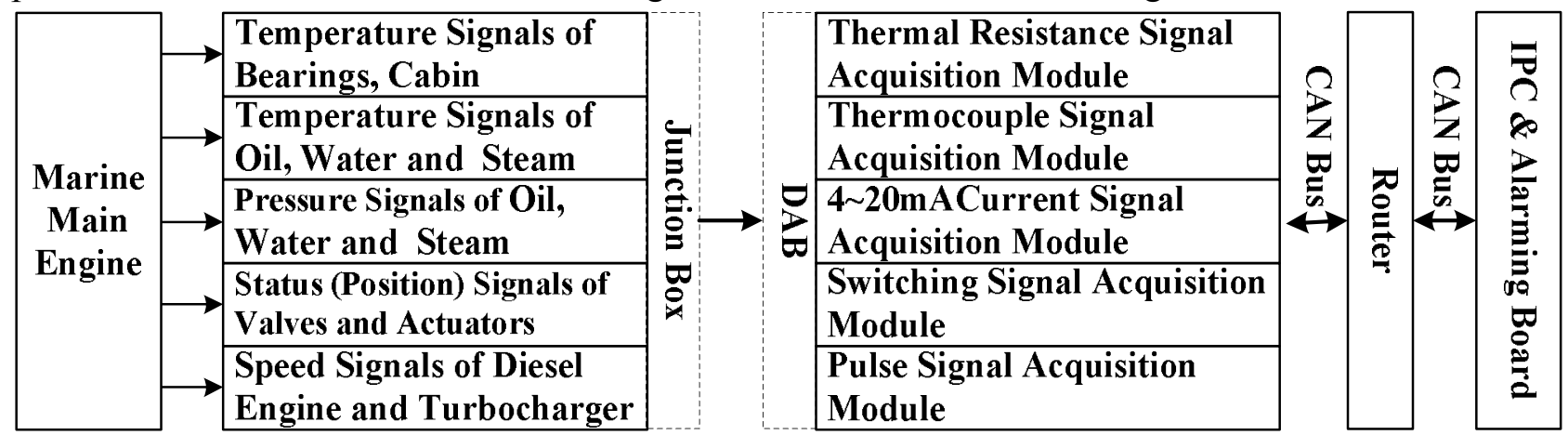

Fig. 4 The Organizational Chart of Remote Control Sub-system and Safety Protection Sub-system 


\section{Integration of RCMS based on inherent testability}

As a new hot field of automatic control, Fieldbus Control System achieves distributed monitoring by integrating field sensors, actuators, servers and network bridge into an entire monitoring system by fieldbus technology, which is the mainstream and direction for the control system [5] . As shown in Fig. 2, this paper use CAN bus to combine every component of RCMS for an effective integration and control system. The whole system is composed of double tiers of CAN network. The upper-level net is for the function of data exchange as well as alarm display, and the latter one provides network support for monitoring and alarm system. In order to ensure the reliability of the system, CAN bus with dual redundant hot standby configuration is constructed.

\section{Conclusions}

The long-term application of RCMS for ship main power plant has proved that the monitoring and control system based on inherent testability design is of great sensitivity, accuracy and reliability, which has made customers satisfactory.

(1) The characteristic of relatively independent configuration for remote control sub-system, safety protection sub-system as well as monitoring and alarm sub-system makes the structure simple and clear, which is benefit for distributed control and centralized management. and it also has strong dangerous dispersion and resistant to damage.

(2) Using single-chip microcomputer control technology and dual redundant CAN Network Communication, the key components are designed with standardized, modularity and universal, make the entire system miniature.

(3) The monitoring system which has the typical structure of alternative combinations is able to satisfy different requirements for ship types and networking models. The system not only can be used for dual-propellers diesel power plant, but also be extended to a four engines and dual-propellers of diesel - diesel combined power plant.

\section{References}

[1] J. Qiu, G.j. Liu and P. Yang etc . Modeling and designing of testability technology for equipment, Science Press, Beijing, 2012. ( In Chinese)

[2] Department of Defense, MIL-HDBK-2165, Military Standard Testability Program for Systems and Equipments, 1995.

[3] GJB 2547A-2012 General requirements of testability for equipment. National Defense Military Standard Press, Beijing, 2012.( In Chinese)

[4] M.y. Zheng. Development of monitoring device for marine diesel engine. Wuhan University of Technology, Wuhan, (2011). ( In Chinese)

[5] P. Oborski. Developments in integration of advanced monitoring systems. The international Journal of Advanced Manufacturing Technology. 75, (2014 ) 1613-1632. 